Rev. Inst. Flor. v. 30 n. 1 p. 53-70 jun. 2018

http://dx.doi.org/10.24278/2178-5031.201830105

ISSN impresso 0103-2674/on-line 2178-5031

\title{
VASCULAR FLORA CHECKLIST OF THE IBICATU ECOLOGICAL STATION, PIRACICABA, SÃO PAULO, BRAZIL ${ }^{1}$
}

\section{ESPÉCIES DA FLORA VASCULAR DA ESTAÇÃO ECOLÓGICA DE IBICATU, PIRACICABA, SÃO PAULO, BRASIL}

\author{
Laíne Silveira CORREAA ${ }^{2,6}$; André Vito SCATIGNA²; Danilo Soares GISSI ${ }^{3}$; Danielle Muniz da SILVA²; \\ Matheus Martins Teixeira COTA ${ }^{4}$; Vinícius Castro SOUZA ${ }^{2,3}$; Natália Macedo IVANAUSKAS; \\ Jorge Yioshio TAMASHIRO2; Ricardo Ribeiro RODRIGUES ${ }^{2,3}$
}

\begin{abstract}
Small forest fragments are recognized as relevant for the preservation of the São Paulo State Semideciduous Seasonal Forest. Our research presents an updated checklist of the vascular flora of the small fragment of legally protected forest, located in the São Paulo State countryside, known as Ibicatu Ecological Station, with the main goal of discussing its importance for local and regional biodiversity conservation. We used the walking method, for a qualitative floristic survey, and botanical material was collected in reproductive and vegetative state. We sampled 187 species typical of the semideciduous seasonal forest, distributed in different eight life forms, with predominance of non-woody life forms, especially herbs and epiphytes. We identified 11 endangered species, including Peperomia hydrocotyloides, considered extinct from the São Paulo State, and some exotic and invasive species. We consider that this small forest fragment present rich vascular flora typical of the São Paulo countryside, in different life forms, and it is essential for local and regional biodiversity. This study added 102 species to the previously published checklist, emphasizing the importance of floristic surveys that include all life forms.
\end{abstract}

Keywords: Atlantic Forest; conservation; Seasonal Semideciduous Forest.

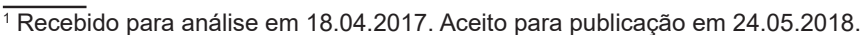

2 Universidade Estadual de Campinas, Instituto de Biologia, Programa de Pós-graduação em Biologia Vegetal, Rua Monteiro Lobato, 255, CEP 13083-862. Campinas, SP, Brasil.

${ }^{3}$ Escola Superior de Agricultura "Luiz de Queiroz", Departamento de Ciências Biológicas, Avenida Pádua Dias, 11, CEP 13418-900, Piracicaba, SP, Brasil.

${ }^{4}$ Universidade de São Paulo, Instituto de Biociências, Departamento de Botânica, Rua do Matão, 277. CEP 05508-090, São Paulo, SP. Brasil.

${ }^{5}$ Instituto Florestal, Divisão de Dasonomia, Seção de Ecologia Florestal, Rua do Horto, 931, Horto Florestal, CEP 02377-000, São Paulo, SP. Brasil.

${ }^{6}$ Autor para correspondência: Laíne Silveira Corrêa - laine06correa@gmail.com
} 


\begin{abstract}
RESUMO - Os pequenos fragmentos florestais são reconhecidamente relevantes para a preservação da Floresta Estacional Semidecidual do estado de São Paulo. Nossa pesquisa apresentou uma ampla lista de espécies da flora vascular de um pequeno fragmento florestal legalmente protegido. Este fragmento localiza-se no interior do estado de São Paulo, e é denominado "Estação Ecológica Ibicatu". Este estudo teve como objetivo principal discutir a importância deste fragmento para a conservação das biodiversidades local e regional. Foi utilizado o método de caminhamento, para um levantamento florístico qualitativo, em que os materiais em estado reprodutivo e vegetativo foram coletados. Foram amostradas 187 espécies típicas da Floresta Estacional Semidecidual, distribuídas em oito distintas formas de vida, com predomínio de formas de vida não lenhosa, e com destaque para ervas e epífitas. Identificaram-se 11 espécies ameaçadas de extinção, dentre elas, Peperomia hydrocotyloides, considerada extinta no estado de São Paulo, assim como algumas espécies exóticas invasoras. Considera-se que este pequeno fragmento florestal mantém uma rica flora vascular típica das florestas do interior paulista, em distintas formas de vida; e por este motivo, relevante para a biodiversidade local e regional. Este estudo também acrescentou 102 espécies à lista anteriormente publicada, enfatizando a importância dos levantamentos florísticos que incluem todas as formas de vida.
\end{abstract}

Palavras-chave: Floresta Atlântica; conservação; Floresta Estacional Semidecidual.

\section{INTRODUCTION}

Tropical forest fragments inserted in cultivated landscapes are, in most cases, small, and undergo degradation, such as the edge effects (Benitez-Malvido and Martinez-Ramos, 2003; Laurence et al., 2007), in addition to other ever-present pressures. Small forest fragments in agricultural landscapes contribute to maintain local fauna (Dias et al., 2016; Beca et al., 2017), ecosystem services (Turner et al., 2007), and can to maintain genetic flow (Martins et al., 2016), among other positive factors for the local and regional biodiversity. It is a key element for the qualitative recovery of human-modified landscapes, to ensure sustainability and improve quality of life (Viana and Pinheiro, 1998; Vidal et al., 2016).

The Atlantic Forest of the São Paulo State countryside, considered a global hotspot for conservation hotspot (Myers, 2003; Mittermeier et al., 2004), has been getting smaller, with more threatened fragments, with only $17.5 \%$ of original forest coverage in the State (São Paulo, 2016a). Even so, many studies carried out in small altered forest fragments (Ivanauskas et al., 1999; Cardoso-Leite and Rodrigues, 2008; Corrêa et al., 2014; Coelho et al., 2016) found local richness in species and its importance for regional species conservation, when considered together with other fragments (Brancalion et al., 2015; Vidal et al., 2016).
The city of Piracicaba, a typical, highly agricultural landscape in central São Paulo State, suffered even more intense devastation, with only $9.2 \%$ remaining of its original vegetation, composed of small fragments (São Paulo, 2016a) with different phytogeographic units such as Seasonal Semideciduous Forest - SSF, Swamp Forest, Seasonal Deciduous Forest and several types of Savanna (Rodrigues, 1999).

Given the relevance of small forest fragments for São Paulo State SSF preservation, we have chosen to expand the knowledge about the Ibicatu Ecological Station floristic composition, a small protected area near Piracicaba. The main goal of our research is to discuss the importance of this small fragment of legally protected forest for local and regional biodiversity conservation.

Thereby, we present an update from previously published checklists of vascular flora (Custódio-Filho et al., 1994; Costa and Mantovani, 1995), which refers only tree and shrub vegetation Our update includes sampling of side ferns, herbs, epiphytes and climber plants, and unpublished tree and shrub species that are present in the Ibicatu Ecological Station. In addition, we verified the endangered species status and identified invasive species. We hope that this checklist will contribute to increase the knowledge about the vascular flora of this small fragment and help establish protection and management policies. 


\section{MATERIALS AND METHODS}

\subsection{Study site}

The Ibicatu Ecological Station is a protected area with 76.40 ha, owned by the Forest Institute of São Paulo, but under management of the Forestry Foundation (São Paulo, 2017). It is in the rural area of Piracicaba, state of São Paulo, between coordinates $22^{\circ} 46^{\prime} 49^{\prime \prime} \mathrm{S}$ and $47^{\circ} 49^{\prime} 09^{\prime \prime} \mathrm{W}$, and elevation between 540-580 meters (Piracicaba, 2017). It is located in a Peripheral Depression in the state of São Paulo, classified as a Seasonal Semideciduous Forest (IBGE, 2012). According to Köeppen (1948), the climate is classified as Cwa (subtropical hot summer climate), with highest average temperatures of $23.9^{\circ} \mathrm{C}$ (January) and lowest of $16.1^{\circ} \mathrm{C}$ (June) (Custódio-Filho et al., 1994).

\subsection{Data collection}

The floristic survey was based on the walking method (Filgueiras et al., 1994), which a quick survey of unpublished species (Walter and Guarino, 2006), to complement the previous protected area checklist to make information easily available to decision-makers (Walter and Guarino, 2006). Botanical material was collected in reproductive and vegetative state by a flora research group, in a three-day expedition carried out in July 2015. We observed other floristic-survey information in the literature mentioned (Leão, 1994; Custódio-Filho et al., 1994; Costa and Mantovani, 1995), which used the plot method to collect quantitative data in this protected area. Our study also included digital records of exsiccates collected in the protected area, available at the SpeciesLink Databases (2016). All collected material was herborized according to IBGE (2012) protocols and incorporated to the ESA Herbarium (acronyms followed Thiers, continuously updated), Piracicaba, and duplicates were sent to UEC and SPSF.

\subsection{Data analysis}

The plants were identified using specialized literature, such as that from the São Paulo State Phanerogamic Flora (Wanderley et al., 2002, 2003, 2005), by comparison with herbaria collections (ESA, SPF and UEC) and Flora and Fungi Virtual Herbarium (INCT, 2016) and consulting experts. The classification of plant families followed APG IV
(2016) for angiosperms and Smith et al. (2006) for ferns. The names were confirmed according to the Missouri Botanical Garden (Tropicos, 2016) and the Brazilian Flora Species List (Flora of Brazil 2020). Conservation status of taxa collected followed the Brazilian Flora Red Book (Martinelli and Moraes, 2013), the list of endangered species of the state of São Paulo (São Paulo, 2016b) and the International Union for Conservation of Nature red list of threatened species (IUCN, 2015). Life form descriptions were defined according to the Brazilian Flora Species List (Flora of Brazil, 2020); exotic plants were considered according to Moro et al. (2012) and Durigan et al. (2013); invasive exotic species were considered according to Richardson et al. (2000).

\section{RESULTS}

This survey sampled 187 vascular species from 132 genera and 69 vascular families in the Ibicatu Ecological Station. From this primary data, we compiled secondary data about the protected area (Custódio-Filho et al., 1994; Costa and Mantovani, 1995; speciesLink, 2016), in a total of 289 species in all life forms (Table 1). Among taxa sampled in the primary data, 23 were fern species and 164 angiosperms species, with nine morpho species. Identification up to the species level found eight life forms (Table 1); however, some species may have two or more life forms (Flora of Brazil, 2020). Non-woody life forms represented $62 \%$ of the survey, especially herbs and epiphytes (Figure 1). Shrubs represented 11\% and trees $27 \%$ of the survey.

Aspleniaceae and Pteridaceae were most represented fern families, both with seven species. Angiosperm families represent $88 \%$ of survey species, but few families represent many species, with Fabaceae (15), Euphorbiaceae (nine), Malvaceae and Meliaceae (eight), Asteraceae and Solanaceae (seven), Rubiaceae and Rutaceae (six), Sapindaceae and Piperaceae (five).

Few genera presented more than one species among all life forms. For non-woody life forms, the climber genera Dalechampia and Serjania, herb genera Adiantum, Doryopteris and Wissadula presented two species. Asplenium and Tillandsia were the most diverse epiphyte genera, with five and three species, respectively. Among tree and shrub life forms, Trichilia (five) and Psychotria (three) were the genera with more species. 
CORREA, L.S. et al. Ibicatu vascular flora.

Table 1. Ibicatu Ecological Station vascular plant species list. H - Habits: Climber (C); Epiphyte herb (EH); Herb (H); Palm (P); Shrub (S); Subshrub (SS); Tree (T); Treelet (TL). RLP - Red lists presence: São Paulo State list (SP - São Paulo, 2016b), Brazilian list (BR - Martinelli and Moraes, 2013) and Global List (GL - International Union for Conservation of Nature red list of threatened species, 2016). Categories of endangered species: Endangered (EN), Vulnerable (VU), Least Concerned (LC). IS - Information source: Primary data (P); Secondary data (S): * Based on survey results by Custódio-Filho and contributors (1994); ** Based on survey results by Costa \& Mantovani (1995). VOUCHER: voucher herbarium specimen (NY, SP or SPSF Herbarium or collector number in ESA Herbarium with duplicates in UEC and SPSF); (1) Brazilian alien species; (2) Brazilian aggressive invader plants.

Tabela 1. Lista de espécies vasculares da Estação Ecológica de Ibicatu. H - Hábito: Trepadeira (C); Epífita Herbácea (EH); Herbácea (H); Palmeira (P); Arbusto (S); Subarbusto (SS); Árvore (T); Arvoreta (TL). RLP - Presença das Espécies em Listas Oficiais de Espécies Ameaçadas: Estado de São Paulo (SP - São Paulo, 2016b), Brasil (BR - Martinelli and Moraes, 2013) e Lista Oficial Internacional (GL - International Union for Conservation of Nature red list of threatened species, 2016). Categorias de Ameaça: Em Perigo (EN); Vulnerável (VU); Menor preocupação (LC). IS - Fonte de informação: Dados primários $(\mathrm{P})$; Dados secundários $(\mathrm{S})$ : * Baseado nos resultados do levantamento de Custódio-Filho e colaboradores (1994); ** Baseado nos resultados do levantamento de Costa \& Mantovani (1995). VOUCHER: espécimes depositadas em herbários (Herbários de NY, SP, SPSF, ou número do coletor no herbário ESA, com duplicatas na UEC e SPSF); (1) espécies exóticas; (2) espécies invasoras e agressivas.

\begin{tabular}{|c|c|c|c|c|}
\hline FAMILY/SPECIES & $\mathrm{H}$ & RLS & IS & VOUCHER \\
\hline \multicolumn{5}{|l|}{ FERNS } \\
\hline \multicolumn{5}{|l|}{ Anemiaceae } \\
\hline Anemia phyllitidis (L.) Sw. & $\mathrm{H}$ & - & $\mathrm{P}$ & Toledo, C.A.P. et al. 104 \\
\hline \multicolumn{5}{|l|}{ Aspleniaceae } \\
\hline Asplenium auritum $\mathrm{Sw}$. & $\mathrm{EH}$ & - & $\mathrm{P}$ & Barroso, R.M. et al. 234 \\
\hline Asplenium bradei Rosenst. & $\mathrm{EH}$ & - & $\mathrm{P}$ & Toledo, C.A.P. et al. 173 \\
\hline Asplenium claussenii Hieron & $\mathrm{EH}$ & - & $\mathrm{P}$ & Barroso, R.M. et al. 230 \\
\hline Asplenium formosum Willd. & $\mathrm{EH}$ & $\mathrm{LC}(\mathrm{GL})$ & $\mathrm{P}$ & Barroso, R.M. et al. 228 \\
\hline Asplenium stuebelianum Hieron & $\mathrm{H}$ & - & $\mathrm{P}$ & Orlandini, P. et al. 149 \\
\hline Asplenium sp. & $\mathrm{EH}$ & - & $\mathrm{P}$ & Barroso, R.M. et al. 229 \\
\hline Hymenasplenium laetum (Sw.) L. Regalado \& Prada & $\mathrm{H}$ & - & $\mathrm{P}$ & Scatigna, A.V. et al. 991 \\
\hline \multicolumn{5}{|l|}{ Dryopteridaceae } \\
\hline Ctenitis sp. & $\mathrm{H}$ & - & $\mathrm{P}$ & Scatigna, A.V. et al. 989 \\
\hline Parapolystichum effusum (Sw.) Ching & $\mathrm{H}$ & - & $\mathrm{P}$ & Scatigna, A.V. et al. 992 \\
\hline \multicolumn{5}{|l|}{ Hymenophyllaceae } \\
\hline Didymoglossum reptans (Sw.) C.Presl & $\mathrm{EH}$ & - & $\mathrm{P}$ & Scatigna, A.V. et al. 987 \\
\hline \multicolumn{5}{|l|}{ Polypodiaceae } \\
\hline Microgramma squamulosa (Kaulf.) de la Sota & $\mathrm{EH}$ & - & $\mathrm{P}$ & Orlandini, P. et al., 101 \\
\hline Pleopeltis minima (Bory) J. Prado \& R.Y. Hirai & $\mathrm{EH}$ & - & $\mathrm{P}$ & Orlandini, P. et al. 112 \\
\hline Pleopeltis pleopeltifolia (Raddi) Alston & $\mathrm{EH}$ & - & $\mathrm{P}$ & Barroso, R.M. et al. 227 \\
\hline \multicolumn{5}{|l|}{ Pteridaceae } \\
\hline Adiantopsis radiata (L.) Fée & $\mathrm{H}$ & - & $\mathrm{P}$ & Toledo, C.A.P. et al. 177 \\
\hline Adiantum diogoanum Glaziou ex. Baker & $\mathrm{H}$ & - & $\mathrm{P}$ & Toledo, C.A.P. et al. 180 \\
\hline Adiantum raddianum $\mathrm{C}$. Presl. & $\mathrm{H}$ & - & $\mathrm{P}$ & Scatigna, A.V. et al. 986 \\
\hline Doryopteris concolor (Langsd. \& Fisch.) Kuhn & $\mathrm{H}$ & - & $\mathrm{P}$ & Scatigna, A.V. et al. 878 \\
\hline Doryopteris pentagona Pic.Serm. & $\mathrm{H}$ & - & $\mathrm{P}$ & Toledo, C.A.P. et al. 105 \\
\hline Hemionitis tomentosa (Lam.) Raddi & $\mathrm{H}$ & - & $\mathrm{P}$ & Toledo, C.A.P. et al. 175 \\
\hline Pteris denticulata Sw. & $\mathrm{H}$ & - & $\mathrm{P}$ & Scatigna, A.V. et al. 875 \\
\hline \multicolumn{5}{|l|}{ Thelypteridaceae } \\
\hline Ciclosorus dentatus (Forssk.) Ching & $\mathrm{H}$ & - & $\mathrm{P}$ & Scatinga, A.V. et al. 887 \\
\hline Macrothelypteris torresiana (Gaudich.) Ching & $\mathrm{H}$ & - & $\mathrm{P}$ & Toledo, C.A.P. et al. 114 \\
\hline
\end{tabular}

to be continued continua 
CORREA, L.S. et al. Ibicatu vascular flora.

continuation - Table 1

continuação - Tabela 1

\begin{tabular}{|c|c|c|c|c|}
\hline FAMILY/SPECIES & $\mathrm{H}$ & RLS & IS & VOUCHER \\
\hline \multicolumn{5}{|l|}{ ANGIOSPERMS } \\
\hline \multicolumn{5}{|l|}{ Acanthaceae } \\
\hline Aphelandra schottiana (Nees) Profice & $\mathrm{H}, \mathrm{S}$ & - & $\mathrm{P}$ & Scatigna, A.V. et al. 876 \\
\hline Justicia lythroides (Nees) V.A.W.Graham & $\mathrm{H}$ & - & $\mathrm{P}$ & Scatigna, A.V. et al. 869 \\
\hline Ruellia brevifolia (Pohl) C.Ezcurra & $\mathrm{SS}$ & - & $\mathrm{P}$ & Scatigna, A.V. et al. 874 \\
\hline \multicolumn{5}{|l|}{ Amaranthaceae } \\
\hline Alternanthera tenella Colla & $\mathrm{H}$ & - & $\mathrm{P}$ & Scatigna, A.V. et al. 883 \\
\hline \multicolumn{5}{|l|}{ Anacardiaceae } \\
\hline Astronium graveolens Jacq. & $\mathrm{T}$ & - & $\mathrm{P}$ & Orlandini, P. et al. 135 \\
\hline Lithrea molleoides (Vell.) Engl. & $\mathrm{T}$ & - & $\mathrm{P}$ & Orlandini, P. et al. 246 \\
\hline Tapirira guianensis Aubl. & $\mathrm{T}$ & - & $\mathrm{S}(*)$ & \\
\hline \multicolumn{5}{|l|}{ Annonaceae } \\
\hline Annona dolabripetala Raddi & $\mathrm{T}$ & - & $\mathrm{P}$ & Orlandini, P. et al. 131 \\
\hline Annona parviflora (A.St.-Hil.) H.Rainer & $\mathrm{T}$ & - & $\mathrm{S}(*)$ & \\
\hline Guatteria australis A.St.-Hil. & $\mathrm{TL}, \mathrm{T}$ & - & $\mathrm{S}(* *)$ & \\
\hline \multicolumn{5}{|l|}{ Apocynaceae } \\
\hline Aspidosperma cylindrocarpon Müll.Arg. & $\mathrm{T}$ & - & $\mathrm{S}(* *)$ & \\
\hline Aspidosperma olivaceum Müll.Arg. & $\mathrm{T}$ & - & $\mathrm{S}(* *)$ & \\
\hline Aspidosperma polyneuron Müll.Arg. & $\mathrm{T}$ & $\mathrm{EN}(\mathrm{GL})$ & $\mathrm{P}$ & Barroso, R.M. et al. 92 \\
\hline Aspidosperma ramiflorum Müll.Arg. & $\mathrm{T}$ & - & $\mathrm{P}$ & Barroso, R.M. et al. 95 \\
\hline Tabernaemontana catharinensis A.DC. & $\mathrm{T}$ & - & $\mathrm{P}$ & Orlandini, P. et al. 233 \\
\hline \multicolumn{5}{|l|}{ Araliaceae } \\
\hline Dendropanax cuneatus (DC.) Dcne. \& Planch. & $\mathrm{T}$ & - & $\mathrm{S}(*)$ & \\
\hline \multicolumn{5}{|l|}{ Arecaceae } \\
\hline Acrocomia aculeata (Jacq.) Lodd. ex Mart. & $\mathrm{P}$ & - & $\mathrm{P}$ & Scatigna, A.V. et al. 988 \\
\hline Euterpe edulis Mart. & $\mathrm{P}$ & $\begin{array}{l}\text { VU(SP), } \\
\text { EN(BR) }\end{array}$ & $\mathrm{P}$ & Orlandini, P. et al. 251 \\
\hline Syagrus romanzoffiana (Cham.) Glassman & $\mathrm{P}$ & - & $\mathrm{S}(*)$ & \\
\hline \multicolumn{5}{|l|}{ Asteraceae } \\
\hline $\begin{array}{l}\text { Chromolaena maximilianii (Schrad. ex DC.) R.M.King } \\
\text { \& H.Rob. }\end{array}$ & $\mathrm{H}$ & - & $\mathrm{P}$ & Scatigna, A.V. et al. 882 \\
\hline Chromolaena sp. & $\mathrm{H}$ & - & $\mathrm{P}$ & Scatigna, A.V. et al. 971 \\
\hline Mikania glomerata Spreng. & $\mathrm{C}$ & - & $\mathrm{P}$ & Barroso, R.M. et al. 223 \\
\hline Moquiniastrum polymorphum (Less.) G. Sancho & $\mathrm{T}$ & - & $\mathrm{P}$ & Orlandini, P. et al. 145 \\
\hline Piptocarpha axillaris (Less.) Baker & $\mathrm{T}$ & - & $\mathrm{S}(*)$ & \\
\hline Sphagneticola trilobata (L.) Pruski & $\mathrm{H}$ & - & $\mathrm{P}$ & Scatigna, A.V. et al. 881 \\
\hline Synedrela nodiflora (L.) Gaertn. & $\mathrm{H}$ & - & $\mathrm{P}$ & Scatigna, A.V. et al. 868 \\
\hline Vernonanthura brasiliana (L.) H.Rob. & $\mathrm{H}$ & - & $\mathrm{P}$ & Toledo, C.A.P. et al. 113 \\
\hline Vernonanthura puberula (Less.) H.Rob. & $\mathrm{T}$ & - & $\mathrm{S}(*)$ & \\
\hline \multicolumn{5}{|l|}{ Balanoforaceae } \\
\hline Scybalium fungiforme Schott \& Endl. & $\mathrm{H}$ & - & $\mathrm{P}$ & Toledo, C.A.P. et al. 184 \\
\hline \multicolumn{5}{|l|}{ Bignoniaceae } \\
\hline Handroanthus albus (Cham.) Mattos & $\mathrm{T}$ & - & $\mathrm{S}(* *)$ & \\
\hline Handroanthus ochraceus (Cham.) Mattos & $\mathrm{T}$ & - & $\mathrm{P}$ & Barroso, R.M. et al. 113 \\
\hline Jacaranda micrantha Cham. & $\mathrm{T}$ & - & $\mathrm{S}(*)$ & \\
\hline Jacaranda puberula Cham. & $\mathrm{T}$ & - & $\mathrm{S}(* *)$ & \\
\hline
\end{tabular}

to be continued continua 
CORRÊA, L.S. et al. Ibicatu vascular flora.

continuation - Table 1

continuação - Tabela 1

\begin{tabular}{|c|c|c|c|c|}
\hline FAMILY/SPECIES & $\mathrm{H}$ & RLS & IS & VOUCHER \\
\hline Tecoma stans (L.) Juss. ex Kunth ${ }^{(1)}$ & $\mathrm{T}$ & - & $\mathrm{P}$ & Barroso, R.M. et al. 112 \\
\hline Indeterminate & $\mathrm{C}$ & - & $\mathrm{P}$ & Orlandini, P. et al. 102 \\
\hline \multicolumn{5}{|l|}{ Boraginaceae } \\
\hline Cordia americana (L.) Gottschling \& J.S.Mill. & $\mathrm{T}$ & - & $\mathrm{P}$ & Barroso, R.M. et al. 84 \\
\hline Cordia ecalyculata Vell. & $\mathrm{T}$ & - & $\mathrm{P}$ & Orlandini, P. et al. 231 \\
\hline Cordia sellowiana Cham. & $\mathrm{T}$ & - & $\mathrm{S}(*)$ & \\
\hline Cordia trichotoma (Vell.) Arráb. ex Steud. & $\mathrm{T}$ & - & $\mathrm{S}(*)$ & \\
\hline Heliotropium transalpinum Vell. & $\mathrm{S}$ & - & $\mathrm{P}$ & Toledo, C.A.P. et al. 94 \\
\hline \multicolumn{5}{|l|}{ Bromeliaceae } \\
\hline $\begin{array}{l}\text { Acanthostachys strobilacea (Schult. \& Schult.f.) } \\
\text { Klotzsch. }\end{array}$ & $\mathrm{EH}$ & - & $\mathrm{S}$ & NY 376272 \\
\hline Aechmea bromeliifolia (Rudge) Baker & $\mathrm{EH}$ & - & $\mathrm{P}$ & Scatigna, A.V. et al. 898 \\
\hline Tillandsia recurvata (L.) L. & $\mathrm{EH}$ & - & $\mathrm{P}$ & Orlandini, P. et al. 100 \\
\hline Tillandsia tricholepis Baker & $\mathrm{EH}$ & - & $\mathrm{P}$ & Orlandini, P. et al. 95 \\
\hline Tillandsia usneoides (L.) L. & $\mathrm{EH}$ & - & $\mathrm{P}$ & Orlandini, P. et al. 99 \\
\hline \multicolumn{5}{|l|}{ Cactaceae } \\
\hline Epiphyllum phyllanthus (L.) Haw. & $\mathrm{EH}$ & $\mathrm{LC}(\mathrm{GL})$ & $\mathrm{P}$ & Orlandini, P. et al. 98 \\
\hline Rhipsalis cereuscula Haw. & $\mathrm{EH}$ & $\mathrm{LC}(\mathrm{GL})$ & $\mathrm{P}$ & Barroso, R.M. et al. 224 \\
\hline \multicolumn{5}{|l|}{ Cannabaceae } \\
\hline Celtis iguanaea (Jacq.) Sarg. & $\mathrm{S}$ & - & $\mathrm{P}$ & Barroso, R.M. et al. 125 \\
\hline \multicolumn{5}{|l|}{ Cardiopteridaceae } \\
\hline \multicolumn{4}{|l|}{ Caricaceae } & \\
\hline Jacaratia spinosa (Aubl.) A.DC. & $\mathrm{T}$ & - & $\mathrm{P}$ & Barroso, R.M. et al. 101 \\
\hline Vasconcellea quercifolia A.St.-Hil. & $\mathrm{TL}, \mathrm{T}$ & - & $\mathrm{S}(* *)$ & \\
\hline \multicolumn{5}{|l|}{ Celastraceae } \\
\hline Maytenus aquifolia Mart. & $\mathrm{T}$ & - & $\mathrm{P}$ & Barroso, R.M. et al. 90 \\
\hline Maytenus cestrifolia Reissek & $\mathrm{TL}, \mathrm{T}$ & - & $\mathrm{S}(* *)$ & \\
\hline Maytenus gonoclada Mart. & $\mathrm{T}$ & - & $\mathrm{S}(*)$ & \\
\hline Maytenus ilicifolia Mart. ex Reissek & $\mathrm{T}$ & VU(SP) & $\mathrm{P}$ & Orlandini, P. et al. 143 \\
\hline \multicolumn{5}{|l|}{ Commelinaceae } \\
\hline Commelina obliqua Vahl & $\mathrm{H}$ & - & $\mathrm{P}$ & Scatigna, A.V. et al. 897 \\
\hline Tradescantia zanonia (L.) Sw. & $\mathrm{H}$ & - & $\mathrm{P}$ & Toledo, C.A.P. et al. 178 \\
\hline Tradescantia zebrina Heynh. ex Bosse (2) & $\mathrm{H}$ & - & $\mathrm{P}$ & Scatigna, A.V. et al. 870 \\
\hline \multicolumn{5}{|l|}{ Convolvulaceae } \\
\hline Ipomoea ramosissima (Poir.) Choisy & $\mathrm{H}$ & - & $\mathrm{P}$ & Toledo, C.A.P. et al. 108 \\
\hline \multicolumn{5}{|l|}{ Cyperaceae } \\
\hline Cyperus iria L. & $\mathrm{H}$ & $\mathrm{LC}(\mathrm{GL})$ & $\mathrm{P}$ & Scatigna, A.V. et al. 880 \\
\hline Scleria gaertneri Raddi & $\mathrm{H}$ & - & $\mathrm{P}$ & Scatigna, A.V. et al. 890 \\
\hline \multicolumn{5}{|l|}{ Dioscoreaceae } \\
\hline \multicolumn{5}{|l|}{ Euphorbiaceae } \\
\hline Actinostemon concepcionis (Chodat \& Hassl.) Hochr. & $\mathrm{T}$ & - & $\mathrm{S}(*)$ & \\
\hline Actinostemon concolor (Spreng.) Müll.Arg. & $\mathrm{S}$ & - & $\mathrm{P}$ & Toledo, C.A.P. et al. 92 \\
\hline Actinostemon klotzschii (Didr.) Pax & $\mathrm{S}$ & - & $\mathrm{P}$ & Scatigna, A.V. et al. 968 \\
\hline Alchornea glandulosa Poepp. \& Endl. & $\mathrm{T}$ & - & $\mathrm{S}(*)$ & \\
\hline
\end{tabular}

to be continued continua 
CORRÊA, L.S. et al. Ibicatu vascular flora.

continuation - Table 1

continuação - Tabela 1

\begin{tabular}{|c|c|c|c|c|}
\hline FAMILY/SPECIES & $\mathrm{H}$ & RLS & IS & VOUCHER \\
\hline Alchornea triplinervia (Spreng.) Müll.Arg. & $\mathrm{T}$ & - & $\mathrm{S}(*)$ & \\
\hline Croton floribundus Spreng. & $\mathrm{T}$ & - & $\mathrm{P}$ & Orlandini, P. et al. 137 \\
\hline Croton urucurana Baill. & $\mathrm{T}$ & - & $\mathrm{S}$ & SP 272492 \\
\hline Dalechampia pentaphylla Lam. & $\mathrm{C}$ & - & $\mathrm{P}$ & Orlandini, P. et al. 104 \\
\hline Dalechampia triphylla Lam. & $\mathrm{C}$ & - & $\mathrm{P}$ & Orlandini, P. et al. 105 \\
\hline Euphorbia sciadophila Boiss. & $\mathrm{H}$ & - & $\mathrm{P}$ & Toledo, C.A.P. et al. 103 \\
\hline Sebastiania serrata (Baill. ex Müll.Arg.) Müll.Arg. & $\mathrm{T}$ & - & $\mathrm{P}$ & Orlandini, P. et al. 126 \\
\hline Gymnanthes klotzschiana Müll.Arg. & $\mathrm{T}$ & - & $\mathrm{S}(*)$ & \\
\hline Pachystroma longifolium (Nees) I.M.Johnst. & $\mathrm{T}$ & - & $\mathrm{P}$ & Barroso, R.M. et al. 103 \\
\hline Indeterminate & $\mathrm{S}$ & - & $\mathrm{P}$ & Scatigna, A.V. et al. 984 \\
\hline \multicolumn{5}{|l|}{ Fabaceae } \\
\hline Albizia edwallii (Hoehne) Barneby \& J.W.Grimes & $\mathrm{T}$ & - & $\mathrm{S}(* / * *)$ & \\
\hline Anadenanthera colubrina (Vell.) Brenan & $\mathrm{T}$ & - & $\mathrm{S}(* *)$ & \\
\hline Bauhinia forficata Link & $\mathrm{T}$ & $\mathrm{LC}(\mathrm{GL})$ & $\mathrm{P}$ & Orlandini, P. et al. 242 \\
\hline Calliandra foliolosa Benth. & $\mathrm{T}$ & - & $\mathrm{P}$ & Orlandini, P. et al. 116 \\
\hline Calliandra tweedii Benth. & $\mathrm{S}, \mathrm{T}$ & - & $\mathrm{S}(* *)$ & \\
\hline Cassia ferruginea (Schrad.) Schrad. ex DC. & $\mathrm{T}$ & - & $\mathrm{S}(*)$ & \\
\hline Centrolobium tomentosum Guillem. ex Benth. & $\mathrm{T}$ & - & $\mathrm{P}$ & Orlandini, P. et al. 142 \\
\hline Chamaecrista rotundifolia (Pers.) Greene & $\mathrm{H}$ & - & $\mathrm{P}$ & Toledo, C.A.P. et al. 110 \\
\hline $\begin{array}{l}\text { Dahlstedtia muehlbergiana (Hassl.) M.J.Silva \& } \\
\text { A.M.G. Azevedo }\end{array}$ & $\mathrm{T}$ & - & $\mathrm{S}(*)$ & \\
\hline Dalbergia frutescens (Vell.) Britton & $\mathrm{S}$ & - & $\mathrm{S}(* *)$ & \\
\hline Enterolobium contortisiliquum (Vell.) Morong & $\mathrm{T}$ & - & $\mathrm{S}(*)$ & \\
\hline Holocalyx balansae Micheli & $\mathrm{T}$ & - & $\mathrm{P}$ & Barroso, R.M. et al. 100 \\
\hline Inga edulis Mart. & $\mathrm{T}$ & - & $\mathrm{S}(* *)$ & \\
\hline Inga marginata Willd. & $\mathrm{T}, \mathrm{TL}$ & $\mathrm{LC}(\mathrm{GL})$ & $\mathrm{P}$ & Scatigna, A.V. et al. 977 \\
\hline Inga striata Benth. & $\mathrm{T}$ & $\mathrm{LC}(\mathrm{GL})$ & $\mathrm{S}(*)$ & \\
\hline $\begin{array}{l}\text { Lonchocarpus cultratus (Vell.) A.M.G.Azevedo \& } \\
\text { H.C.Lima }\end{array}$ & $\mathrm{T}$ & - & $\mathrm{P}$ & Orlandini, P. et al. 127 \\
\hline Machaerium brasiliense Vogel & $\mathrm{T}$ & $\mathrm{LC}(\mathrm{GL})$ & $\mathrm{S}(*)$ & \\
\hline Machaerium nyctitans (Vell.) Benth. & $\mathrm{T}$ & - & $\mathrm{P}$ & Orlandini, P. et al. 151 \\
\hline Machaerium scleroxylon Tul. & $\mathrm{T}$ & $\mathrm{LC}(\mathrm{GL})$ & $\mathrm{P}$ & Barroso, R.M. et al. 88 \\
\hline Machaerium stipitatum Vogel & $\mathrm{T}$ & - & $\mathrm{S}(*)$ & \\
\hline Machaerium uncinatum (Vell.) Benth. & $\mathrm{S}$ & - & $\mathrm{S}(* *)$ & \\
\hline Machaerium villosum Vogel & $\mathrm{T}$ & VU (GL) & $\mathrm{S}(*)$ & \\
\hline Myroxylon balsamum (L.) Harms & $\mathrm{T}$ & - & $\mathrm{S}(*)$ & \\
\hline Myroxylon peruiferum L.f. & $\mathrm{T}$ & - & $\mathrm{P}$ & Barroso, R.M. et al. 107 \\
\hline Peltophorum dubium (Spreng.) Taub. & $\mathrm{T}$ & - & $\mathrm{P}$ & Barroso, R.M. et al. 99 \\
\hline Piptadenia gonoacantha (Mart.) J.F.Macbr. & $\mathrm{T}$ & - & $\mathrm{S}(*)$ & \\
\hline Platypodium elegans Vogel & $\mathrm{T}$ & $\mathrm{LC}(\mathrm{GL})$ & $\mathrm{S}(*)$ & \\
\hline Pterocarpus rohrii Vahl & $\mathrm{T}$ & - & $\mathrm{S}(* *)$ & \\
\hline Senegalia polyphylla (DC.) Britton \& Rose & $\mathrm{T}$ & - & $\mathrm{P}$ & Orlandini, P. et al. 234 \\
\hline Senna hirsuta (L.) H.S.Irwin \& Barneby & $\mathrm{S}$ & - & $\mathrm{P}$ & Toledo, C.A.P. et al. 87 \\
\hline $\begin{array}{l}\text { Senna pendula (Humb.\& Bonpl. ex Willd.) H.S.Irwin } \\
\text { \& Barneby }\end{array}$ & $\mathrm{S}$ & $\mathrm{LC}(\mathrm{GL})$ & $\mathrm{P}$ & Barroso, R.M. et al. 124 \\
\hline Zollernia ilicifolia (Brongn.) Vogel & $\mathrm{T}$ & - & $\mathrm{P}$ & Barroso, R.M. et al. 118 \\
\hline
\end{tabular}

to be continued continua 
CORRÊA, L.S. et al. Ibicatu vascular flora.

continuation - Table 1

continuação - Tabela 1

\begin{tabular}{|c|c|c|c|c|}
\hline FAMILY/SPECIES & $\mathrm{H}$ & RLS & IS & VOUCHER \\
\hline \multicolumn{5}{|l|}{ Lamiaceae } \\
\hline Aegiphila integrifolia (Jacq.) Moldenke & $\mathrm{T}$ & - & $\mathrm{P}$ & Orlandini, P. et al. 140 \\
\hline Marsypianthes chamaedrys (Vahl) Kuntze & $\mathrm{H}$ & - & $\mathrm{P}$ & Toledo, C.A.P. et al. 115 \\
\hline Vitex megapotamica (Spreng.) Moldenke & $\mathrm{T}$ & - & $\mathrm{S}(*)$ & \\
\hline Indeterminate & $\mathrm{H}$ & - & $\mathrm{P}$ & Toledo, C.A.P. et al. 107 \\
\hline \multicolumn{5}{|l|}{ Lauraceae } \\
\hline Endlicheria paniculata (Spreng.) J.F.Macbr. & $\mathrm{T}$ & - & $\mathrm{S}(*)$ & \\
\hline Nectandra lanceolata Nees & $\mathrm{T}$ & - & $\mathrm{S}(*)$ & \\
\hline Nectandra megapotamica (Spreng.) Mez & $\mathrm{T}$ & - & $\mathrm{P}$ & Orlandini, P. et al. 250 \\
\hline Nectandra oppositifolia Nees & $\mathrm{T}$ & - & $\mathrm{S}(*)$ & \\
\hline Ocotea corymbosa (Meisn.) Mez & $\mathrm{T}$ & - & $\mathrm{S}(* *)$ & \\
\hline Ocotea elegans $\mathrm{Mez}$ & $\mathrm{T}$ & - & $\mathrm{S}(*)$ & \\
\hline Ocotea lanata (Nees \& Mart.) Mez & $\mathrm{T}$ & - & $\mathrm{S}(* *)$ & \\
\hline Ocotea velloziana (Meisn.) Mez & $\mathrm{T}$ & - & $\mathrm{S}(* *)$ & \\
\hline Ocotea velutina (Nees) Rohwer & $\mathrm{T}$ & - & $\mathrm{S}(*)$ & \\
\hline \multicolumn{5}{|l|}{ Lecythidaceae } \\
\hline Cariniana estrellensis (Raddi) Kuntze & $\mathrm{T}$ & - & $\mathrm{P}$ & Orlandini, P. et al. 124 \\
\hline Cariniana legalis (Mart.) Kuntze & $\mathrm{T}$ & VU (GL) & $\mathrm{P}$ & Barroso, R.M. et al. 108 \\
\hline \multicolumn{5}{|l|}{ Malpighiaceae } \\
\hline Janusia guaranitica (A.St.-Hil.) A.Juss. & $\mathrm{C}$ & - & $\mathrm{P}$ & Scatigna, A.V. et al. 904 \\
\hline Indeterminate & $\mathrm{C}$ & - & $\mathrm{P}$ & Orlandini, P. et al. 109 \\
\hline \multicolumn{5}{|l|}{ Malvaceae } \\
\hline Bastardiopsis densiflora (Hook. \& Arn.) Hassl. & $\mathrm{T}$ & - & $\mathrm{P}$ & Barroso, R.M. et al. 86 \\
\hline Callianthe fluviatilis (Vell.) Donnel & $\mathrm{S}$ & - & $\mathrm{S}(* *)$ & \\
\hline Ceiba speciosa (A.St.-Hil.) Ravenna & $\mathrm{T}$ & - & $\mathrm{P}$ & Barroso, R.M. et al. 102 \\
\hline Eriotheca candolleana (K.Schum.) A.Robyns & $\mathrm{T}$ & - & $\mathrm{S}(*)$ & \\
\hline Guazuma ulmifolia Lam. & $\mathrm{T}$ & - & $\mathrm{P}$ & Orlandini, P. et al. 241 \\
\hline Heliocarpus popayanensis Kunth & $\mathrm{T}$ & - & $\mathrm{S}(*)$ & \\
\hline Luehea divaricata Mart. \& Zucc. & $\mathrm{T}$ & - & $\mathrm{P}$ & Orlandini, P. et al. 240 \\
\hline Sida planicaulis Cav. & $\mathrm{H}, \mathrm{S}$ & - & $\mathrm{P}$ & Scatigna, A.V. et al. 879 \\
\hline Sidastrum micranthum (A.St.-Hil.) Fryxell & $\mathrm{H}$ & - & $\mathrm{P}$ & Toledo, C.A.P. et al. 102 \\
\hline Wissadula hernandioides (L.Hér.) Garcke & $\mathrm{H}$ & - & $\mathrm{P}$ & Scatigna, A.V. et al. 889 \\
\hline Wissadula wissadifolia (Griseb.) Krapov. & $\mathrm{H}$ & - & $\mathrm{P}$ & Toledo, C.A.P. et al. 112 \\
\hline \multicolumn{5}{|l|}{ Melastomataceae } \\
\hline Clidemia hirta (L.) D.Don & $\mathrm{S}$ & - & $\mathrm{P}$ & Scatigna, A.V. et al. 998 \\
\hline Miconia discolor DC. & $\mathrm{S}$ & - & $\mathrm{P}$ & Barroso, R.M. et al. 122 \\
\hline \multicolumn{5}{|l|}{ Meliaceae } \\
\hline Cabralea canjerana (Vell.) Mart. & $\mathrm{T}$ & - & $\mathrm{S}(*)$ & \\
\hline Cedrela fissilis Vell. & $\mathrm{T}$ & $\mathrm{EN}(\mathrm{GL})$ & $\mathrm{P}$ & Orlandini, P. et al. 244 \\
\hline Guarea guidonia (L.) Sleumer & $\mathrm{T}$ & - & $\mathrm{P}$ & Orlandini, P. et al. 247 \\
\hline Guarea macrophylla Vahl & $\mathrm{T}$ & - & $\mathrm{P}$ & Orlandini, P. et al. 133 \\
\hline Trichilia casaretti C.DC. & $\mathrm{T}$ & VU (GL) & $\mathrm{S}(* *)$ & \\
\hline Trichilia catigua A.Juss. & $\mathrm{T}$ & - & $\mathrm{P}$ & Barroso, R.M. et al. 93 \\
\hline Trichilia clausseni C.DC. & $\mathrm{T}$ & - & $\mathrm{P}$ & Barroso, R.M. et al. 94 \\
\hline Trichilia elegans A.Juss. & $\mathrm{T}$ & - & $\mathrm{P}$ & Barroso, R.M. et al. 110 \\
\hline Trichilia pallida $\mathrm{Sw}$. & $\mathrm{T}$ & - & $\mathrm{P}$ & Orlandini, P. et al. 153 \\
\hline Trichilia silvatica C.DC. & $\mathrm{T}$ & VU (GL) & $\mathrm{P}$ & Orlandini, P. et al. 122 \\
\hline
\end{tabular}

to be continued continua 
CORRÊA, L.S. et al. Ibicatu vascular flora.

continuation - Table 1

continuação - Tabela 1

\begin{tabular}{|c|c|c|c|c|}
\hline FAMILY/SPECIES & $\mathrm{H}$ & RLS & IS & VOUCHER \\
\hline \multicolumn{5}{|l|}{ Menispermaceae } \\
\hline Cissampelos pareira $\mathrm{L}$. & $\mathrm{C}$ & VU (SP) & $\mathrm{P}$ & Scatigna, A.V. et al. 900 \\
\hline \multicolumn{5}{|l|}{ Moraceae } \\
\hline Ficus gomelleira Kunth & $\mathrm{T}$ & - & $\mathrm{S}(*)$ & \\
\hline Ficus guaranitica Chodat & $\mathrm{T}$ & - & $\mathrm{P}$ & Orlandini, P. et al. 148 \\
\hline Ficus luschnathiana (Miq.) Miq. & $\mathrm{T}$ & - & $\mathrm{S}(*)$ & \\
\hline Sorocea bonplandii (Baill.) W.C.Burger et al. & $\mathrm{TL}, \mathrm{T}$ & - & $\mathrm{S}(* / * *)$ & \\
\hline \multicolumn{5}{|l|}{ Myrtaceae } \\
\hline Calyptranthes gradiflora O.Berg. & $\mathrm{T}$ & - & $\mathrm{S}(* *)$ & \\
\hline Campomanesia guaviroba (DC.) Kiaersk. & $\mathrm{T}$ & - & $\mathrm{S}(*)$ & \\
\hline Campomanesia guazumifolia (Camb.) O.Berg. & $\mathrm{T}$ & - & $\mathrm{S}(* *)$ & \\
\hline Campomanesia neriiflora (O.Berg) Nied. & $\mathrm{T}$ & VU (GL) & $\mathrm{S}$ & SP 272496 \\
\hline Campomanesia xanthocarpa (Mart.) O.Berg & $\mathrm{T}$ & - & $\mathrm{P}$ & Orlandini, P. et al. 227 \\
\hline Eugenia florida DC. & $\mathrm{T}$ & - & $\mathrm{S}(*)$ & \\
\hline Eugenia sphenophylla O.Berg & $\mathrm{T}$ & - & $\mathrm{S}(* *)$ & \\
\hline Eugenia sulcata Spring ex Mart. & $\mathrm{T}$ & - & $\mathrm{S}(* *)$ & \\
\hline Myrciaria floribunda (H.West ex Willd.) O.Berg & $\mathrm{T}$ & - & $\mathrm{S}(*)$ & \\
\hline Plinia rivularis (Cambess.) Rotman & $\mathrm{T}$ & - & $\mathrm{S}(*)$ & \\
\hline Siphoneugena densiflora O.Berg & $\mathrm{T}$ & - & $\mathrm{S}(* *)$ & \\
\hline Indeterminate & $\mathrm{T}$ & - & $\mathrm{P}$ & Orlandini, P. et al. 141 \\
\hline Indeterminate & $\mathrm{T}$ & - & $\mathrm{P}$ & Orlandini, P. et al. 152 \\
\hline Indeterminate & $\mathrm{T}$ & - & $\mathrm{P}$ & Orlandini, P. et al. 226 \\
\hline \multicolumn{5}{|l|}{ Nyctaginaceae } \\
\hline Guapira opposita (Vell.) Reitz & $\mathrm{T}$ & - & $\mathrm{S}(*)$ & \\
\hline Neea parviflora Poepp. \& Endl. & $\mathrm{TL}, \mathrm{T}$ & - & $\mathrm{S}(* *)$ & \\
\hline Pisonia aculeata $\mathrm{L}$. & $\mathrm{S}, \mathrm{C}$ & - & $\mathrm{P}$ & Scatigna, A.V. et al. 907 \\
\hline \multicolumn{5}{|l|}{ Olacaceae } \\
\hline Heisteria silviani Schwacke & $\mathrm{T}$ & - & $\mathrm{S}(*)$ & \\
\hline \multicolumn{5}{|l|}{ Onagraceae } \\
\hline Ludwigia octovalvis (Benth.) P.H.Raven & $\mathrm{H}$ & LC(GL) & $\mathrm{P}$ & Scatigna, A.V. et al. 892 \\
\hline \multicolumn{5}{|l|}{ Orchidaceae } \\
\hline Cyclopogon variegatus Barb.Rodr. & $\mathrm{H}$ & - & $\mathrm{P}$ & Toledo, C.A.P. et al. 106 \\
\hline Oeceoclades maculata (Lindl.) Lindl. & $\mathrm{H}$ & LC(GL) & $\mathrm{P}$ & Scatigna, A.V. et al. 873 \\
\hline Zeuxine strateumatica (L.) Schltr. ${ }^{(1)}$ & $\mathrm{H}$ & $\mathrm{LC}(\mathrm{GL})$ & $\mathrm{P}$ & Scatigna, A.V. et al. 886 \\
\hline \multicolumn{5}{|l|}{ Oxalidaceae } \\
\hline Oxalis rhombeo-ovata A.St.-Hil. & $\mathrm{SS}, \mathrm{S}, \mathrm{H}$ & - & $\mathrm{P}$ & Scatigna, A.V. et al. 983 \\
\hline \multicolumn{5}{|l|}{ Phyllanthaceae } \\
\hline Savia dictyocarpa Müll.Arg. & $\mathrm{S}, \mathrm{T}$ & - & $\mathrm{P}$ & Orlandini, P. et al. 117 \\
\hline \multicolumn{5}{|l|}{ Phytolaccaceae } \\
\hline Gallesia integrifolia (Spreng.) Harms & $\mathrm{T}$ & - & $\mathrm{P}$ & Barroso, R.M. et al. 96 \\
\hline Seguiera aculeata Jacq. & $\mathrm{S}$ & - & $\mathrm{S}(* *)$ & \\
\hline Seguieria langsdorffii Moq. & $\mathrm{T}$ & - & $\mathrm{P}$ & Barroso, R.M. et al. 109 \\
\hline \multicolumn{5}{|l|}{ Piperaceae } \\
\hline Peperomia delicatula Henschen & $\mathrm{EH}$ & - & $\mathrm{P}$ & Scatigna, A.V. et al. 903 \\
\hline Peperomia hydrocotyloides Miq. & $\mathrm{H}$ & EX (SP) & $\mathrm{P}$ & Scatigna, A.V. et al. 990 \\
\hline Peperomia rotundifolia (L.) Kunth & $\mathrm{EH}$ & - & $\mathrm{P}$ & Barroso, R.M. et al. 231 \\
\hline
\end{tabular}

to be continued continua 
CORREA, L.S. et al. Ibicatu vascular flora.

continuation - Table 1

continuação - Tabela 1

\begin{tabular}{|c|c|c|c|c|}
\hline FAMILY/SPECIES & $\mathrm{H}$ & RLS & IS & VOUCHER \\
\hline Piper amalago $\mathrm{L}$. & $\mathrm{S}$ & - & $\mathrm{P}$ & Barroso, R.M. et al. 119 \\
\hline Piper lhotzkyanum Kunth & $\mathrm{H}, \mathrm{S}$ & - & $\mathrm{P}$ & Scatigna, A.V. et al. 969 \\
\hline \multicolumn{5}{|l|}{ Plantaginaceae } \\
\hline Scoparia dulcis L. & $\mathrm{H}$ & - & $\mathrm{P}$ & Scatigna, A.V. et al. 891 \\
\hline \multicolumn{5}{|l|}{ Poaceae } \\
\hline Acroceras zizanoides (Kunth) Dandy & $\mathrm{H}$ & - & $\mathrm{P}$ & Scatigna, A.V. et al. 888 \\
\hline Plismenus hirtellus (L.) P.Beauv. & $\mathrm{H}$ & - & $\mathrm{P}$ & Scatigna, A.V. et al. 872 \\
\hline Indeterminate & $\mathrm{H}$ & - & $\mathrm{P}$ & Scatigna, A.V. et al. 871 \\
\hline Indeterminate & $\mathrm{H}$ & - & $\mathrm{P}$ & Toledo, C.A.P. et al. 111 \\
\hline \multicolumn{5}{|l|}{ Polygonaceae } \\
\hline Polygonum persicaria $\mathrm{L}$. & $\mathrm{H}$ & $\mathrm{LC}(\mathrm{GL})$ & $\mathrm{P}$ & Scatigna, A.V. et al. 895 \\
\hline Ruprechtia laxiflora Meisn. & $\mathrm{T}$ & - & $\mathrm{P}$ & Barroso, R.M. et al. 91 \\
\hline \multicolumn{5}{|l|}{ Primulaceae } \\
\hline Geissanthus ambiguous (Mart.) G.Agostini & $\mathrm{T}$ & - & $\mathrm{P}$ & Orlandini, P. et al. 123 \\
\hline Myrsine balansae (Mez) Otegui & $\mathrm{T}$ & - & $\mathrm{P}$ & Orlandini, P. et al. 120 \\
\hline Myrsine coriacea (Sw.) R.Br. ex Roem. \& Schult. & $\mathrm{T}$ & - & $\mathrm{S}(*)$ & \\
\hline Myrsine gardneriana A.DC. & $\mathrm{T}$ & - & $\mathrm{S}(*)$ & \\
\hline Myrsine umbellata Mart. & $\mathrm{T}$ & - & $\mathrm{S}(*)$ & \\
\hline \multicolumn{5}{|l|}{ Proteaceae } \\
\hline Roupala montana Aubl. & $\mathrm{S}, \mathrm{T}$ & - & $\mathrm{S}(* / * *)$ & \\
\hline \multicolumn{5}{|l|}{ Rhamnaceae } \\
\hline Colubrina glandulosa Perkins & $\mathrm{T}$ & - & $\mathrm{P}$ & Orlandini, P. et al. 136 \\
\hline Gouania virgata Reissek & $\mathrm{C}$ & - & $\mathrm{P}$ & Orlandini, P. et al. 103 \\
\hline Rhamnidium elaeocarpum Reissek & $\mathrm{T}$ & - & $\mathrm{P}$ & Orlandini, P. et al. 238 \\
\hline \multicolumn{5}{|l|}{ Rosaceae } \\
\hline Prunus myrtifolia (L.) Urb. & $\mathrm{T}$ & - & $\mathrm{S}(*)$ & \\
\hline \multicolumn{5}{|l|}{ Rubiaceae } \\
\hline Amaioua guianensis Aubl. & $\mathrm{S}, \mathrm{T}$ & - & $\mathrm{S}(* *)$ & \\
\hline Chomelia ribesioides Benth. ex A.Gray & $\mathrm{S}, \mathrm{T}$ & - & $\mathrm{S}(* *)$ & \\
\hline Coffea arabica L. (1) & $\mathrm{S}$ & - & $\mathrm{S}(* *)$ & \\
\hline Coutarea hexandra (Jacq.) K.Schum & $\mathrm{T}$ & - & $\mathrm{S}(*)$ & \\
\hline Ixora venulosa Benth. & $\mathrm{T}$ & - & $\mathrm{S}(*)$ & \\
\hline Margaritopsis cephalantha (Müll.Arg.) C.M.Taylor & S & - & $\mathrm{P}$ & Scatigna, A.V. et al. 994 \\
\hline Psychotria carthagenensis Jacq. & S & - & $\mathrm{P}$ & Scatigna, A.V. et al. 993 \\
\hline Psychotria leiocarpa Cham. \& Schltdl. & S & - & $\mathrm{S}(* *)$ & \\
\hline Psychotria myriantha Müll.Arg. & $\mathrm{T}$ & - & $\mathrm{P}$ & Orlandini, P. et al. 222 \\
\hline Psychotria sp. & S & - & $\mathrm{P}$ & Scatigna, A.V. et al. 985 \\
\hline Randia armata (Sw.) DC. & $\mathrm{S}, \mathrm{T}$ & - & $\mathrm{P}$ & Toledo, C.A.P. et al. 89 \\
\hline Rudgea jasminoides (Cham.) Müll.Arg. & $\mathrm{S}, \mathrm{T}$ & - & $\mathrm{P}$ & Scatigna, A.V. et al. 972 \\
\hline \multicolumn{5}{|l|}{ Rutaceae } \\
\hline Balfourodendron riedelianum (Engl.) Engl. & $\mathrm{T}$ & $\mathrm{EN}(\mathrm{GL})$ & $\mathrm{P}$ & Barroso, R.M. et al. 97 \\
\hline $\begin{array}{l}\text { Conchocarpus pentandrus (A.St.-Hil.) Kallunki \& } \\
\text { Pirani }\end{array}$ & $\mathrm{S}, \mathrm{T}$ & - & $\mathrm{P}$ & Barroso, R.M. et al. 111 \\
\hline Esenbeckia febrifuga (A.St.-Hil.) A.Juss.ex Mart. & $\mathrm{T}$ & - & $\mathrm{S}(*)$ & \\
\hline Esenbeckia grandiflora Mart. & $\mathrm{T}$ & - & $\mathrm{S}(*)$ & \\
\hline Esenbeckia leiocarpa Engl. & $\mathrm{T}$ & VU (GL) & $\mathrm{P}$ & Barroso, R.M. et al. 106 \\
\hline
\end{tabular}

to be continued continua 
CORRÊA, L.S. et al. Ibicatu vascular flora.

continuation - Table 1

continuação - Tabela 1

\begin{tabular}{|c|c|c|c|c|}
\hline FAMILY/SPECIES & $\mathrm{H}$ & RLS & IS & VOUCHER \\
\hline Metrodorea nigra A.St.-Hil. & $\mathrm{S}, \mathrm{T}$ & - & $\mathrm{P}$ & Scatigna, A.V. etal. 1001 \\
\hline Pilocarpus pauciflorus A.St.-Hil. & $\mathrm{T}$ & - & $\mathrm{P}$ & Orlandini, P. et al. 237 \\
\hline Pilocarpus pennatifolius Lem. & $\mathrm{T}$ & - & $\mathrm{S}(* *)$ & \\
\hline Zanthoxylum acuminatum (Sw.) Sw. & $\mathrm{T}$ & - & $\mathrm{S}(* *)$ & \\
\hline Zanthoxylum fagara (L.) Sarg. & $\mathrm{S}, \mathrm{T}$ & - & $\mathrm{P}$ & Barroso, R.M. et al. 116 \\
\hline Zanthoxylum rhoifolium Lam. & $\mathrm{T}$ & - & $\mathrm{S}(*)$ & \\
\hline \multicolumn{5}{|l|}{ Salicaceae } \\
\hline Casearia decandra Jacq. & $\mathrm{T}$ & - & $\mathrm{S}(*)$ & \\
\hline Casearia gossypiosperma Briq. & $\mathrm{T}$ & - & $\mathrm{P}$ & Barroso, R.M. et al. 87 \\
\hline Casearia sylvestris Sw. & $\mathrm{T}$ & - & $\mathrm{P}$ & Orlandini, P. et al. 239 \\
\hline Prockia crucis P.Browne ex L. & $\mathrm{T}$ & - & $\mathrm{S}(*)$ & \\
\hline Xylosma pseudosalzmanii Sleumer & $\mathrm{T}$ & - & $\mathrm{P}$ & Barroso, R.M. et al. 105 \\
\hline \multicolumn{5}{|l|}{ Sapindaceae } \\
\hline Cupania vernalis Cambess. & $\mathrm{T}$ & - & $\mathrm{P}$ & Orlandini, P. et al. 134 \\
\hline Diatenopteryx sorbifolia Radlk. & $\mathrm{T}$ & - & $\mathrm{P}$ & Scatigna, A.V. et al. 973 \\
\hline Dodonaea viscosa Jacq. & $\mathrm{T}$ & - & $\mathrm{P}$ & Scatigna, A.V. etal. 1000 \\
\hline Matayba elaeagnoides Radlk. & $\mathrm{T}$ & - & $\mathrm{S}(*)$ & \\
\hline Matayba guianensis Aubl. & $\mathrm{TL}, \mathrm{T}$ & - & $\mathrm{S}(* *)$ & \\
\hline Serjania caracasana (Jacq.) Willd. & $\mathrm{C}$ & - & $\mathrm{P}$ & Scatigna, A.V. et al. 906 \\
\hline $\begin{array}{l}\text { Serjania fuscifolia } \text { Radlk. } \\
\text { Sapotaceae }\end{array}$ & \multicolumn{3}{|c|}{ Sapotaceae } & Scatigna, A.V. et al. 901 \\
\hline $\begin{array}{l}\text { Chrysophyllum gonocarpum (Mart. \& Eichler ex Miq.) } \\
\text { Engl. }\end{array}$ & $\mathrm{T}$ & - & $\mathrm{P}$ & Orlandini, P. et al. 138 \\
\hline $\begin{array}{l}\text { Chrysophyllum marginatum (Hook. \& Arn.) Radlk. } \\
\text { Scrophulariaceae }\end{array}$ & $\mathrm{TL}, \mathrm{T}$ & - & $\mathrm{S}(* *)$ & \\
\hline $\begin{array}{l}\text { Buddleja stachyoides Cham. \& Schltdl. } \\
\text { Simaroubaceae }\end{array}$ & \multicolumn{3}{|c|}{ Simaroubaceae } & Scatigna, A.V. et al. 884 \\
\hline \multicolumn{5}{|l|}{ Siparunaceae } \\
\hline $\begin{array}{l}\text { Siparuna brasiliensis (Spreng.) A.DC. } \\
\text { Smilacaceae }\end{array}$ & $\mathrm{TL}, \mathrm{T}$ & - & $\mathrm{S}(* *)$ & \\
\hline $\begin{array}{l}\text { Smilax campestris Griseb. } \\
\text { Solanaceae }\end{array}$ & \multicolumn{3}{|c|}{ Solanaceae } & Barroso, R.M. et al. 226 \\
\hline Acnistus arborescens (L.) Schltdl. & $\mathrm{T}$ & - & $\mathrm{S}(*)$ & \\
\hline Capsicum recurvatum Witasek & $\mathrm{S}$ & - & $\mathrm{P}$ & Scatigna, A.V. et al. 996 \\
\hline Cestrum mariquitense Kunth & $\mathrm{S}$ & - & $\mathrm{P}$ & Toledo, C.A.P. et al. 100 \\
\hline Cestrum strigilatum Ruiz \& Pav. & $\mathrm{S}$ & - & $\mathrm{P}$ & Scatigna, A.V. et al. 894 \\
\hline Solanum concinnum Schott ex Sendtn. & $\mathrm{S}$ & - & $\mathrm{P}$ & Barroso, R.M. et al. 123 \\
\hline Solanum gnaphalocarpon Vell. & $\mathrm{S}$ & - & $\mathrm{P}$ & Scatigna, A.V. et al. 981 \\
\hline Solanum hirtellum (Spreng.) Hassl. & $\mathrm{C}$ & - & $\mathrm{P}$ & Scatigna, A.V. et al. 902 \\
\hline Solanum megalochiton Mart. & $\mathrm{S}$ & - & $\mathrm{S}(* *)$ & \\
\hline Solanum swartzianum Roem. \& Schult. & $\mathrm{S}$ & - & $\mathrm{P}$ & Barroso, R.M. et al. 115 \\
\hline Solanum variable Mart. & $\mathrm{T}, \mathrm{S}$ & - & $\mathrm{S}$ & NY 781315 \\
\hline \multicolumn{5}{|l|}{ Urticaceae } \\
\hline Boehmeria caudata Sw. & $\mathrm{S}, \mathrm{T}$ & - & $\mathrm{P}$ & Orlandini, P. et al. 139 \\
\hline Cecropia glaziovii Snethl. & $\mathrm{T}$ & - & $\mathrm{S}(* *)$ & \\
\hline
\end{tabular}

to be continued continua 
CORRÊA, L.S. et al. Ibicatu vascular flora.

continuation - Table 1

continuação - Tabela 1

\begin{tabular}{|c|c|c|c|c|}
\hline FAMILY/SPECIES & $\mathrm{H}$ & RLS & IS & VOUCHER \\
\hline Cecropia pachystachya Trécul & $\mathrm{T}$ & - & $\mathrm{S}(*)$ & \\
\hline Urera baccifera (L.) Gaudich. ex Wedd. & $\mathrm{S}$ & - & $\mathrm{P}$ & Toledo, C.A.P. et al. 91 \\
\hline \multicolumn{5}{|l|}{ Verbenaceae } \\
\hline Aloysia virgata (Ruiz \& Pav.) Juss. & $\mathrm{T}$ & - & $\mathrm{S}(*)$ & \\
\hline Citharexylum myrianthum Cham. & $\mathrm{T}$ & - & $\mathrm{P}$ & Orlandini, P. et al. 150 \\
\hline Lantana fucata Lindl. & $\mathrm{H}$ & - & $\mathrm{P}$ & Scatigna, A.V. et al. 896 \\
\hline Lantana trifolia $\mathrm{L}$. & SS, $S$ & - & $\mathrm{P}$ & Scatigna, A.V. et al. 885 \\
\hline Petrea volubilis $\mathrm{L}$. & $\mathrm{C}$ & - & $\mathrm{P}$ & Scatigna, A.V. et al. 899 \\
\hline \multicolumn{5}{|l|}{ Violaceae } \\
\hline Pombalia atropurpurea (A.St.-Hil.) Paula-Souza & $\mathrm{S}$ & - & $\mathrm{P}$ & Barroso, R.M. et al. 121 \\
\hline Pombalia bigibbosa (A.St.-Hil.) Paula-Souza & $\mathrm{T}$ & - & $\mathrm{P}$ & Orlandini, P. et al. 125 \\
\hline \multicolumn{5}{|l|}{ Vochysiaceae } \\
\hline Qualea cinnamomea Pohl & $\mathrm{T}$ & - & $\mathrm{P}$ & SPSF 04541 \\
\hline Qualea multiflora Mart. & $\mathrm{T}$ & - & $\mathrm{S}\left({ }^{*}\right)$ & \\
\hline
\end{tabular}

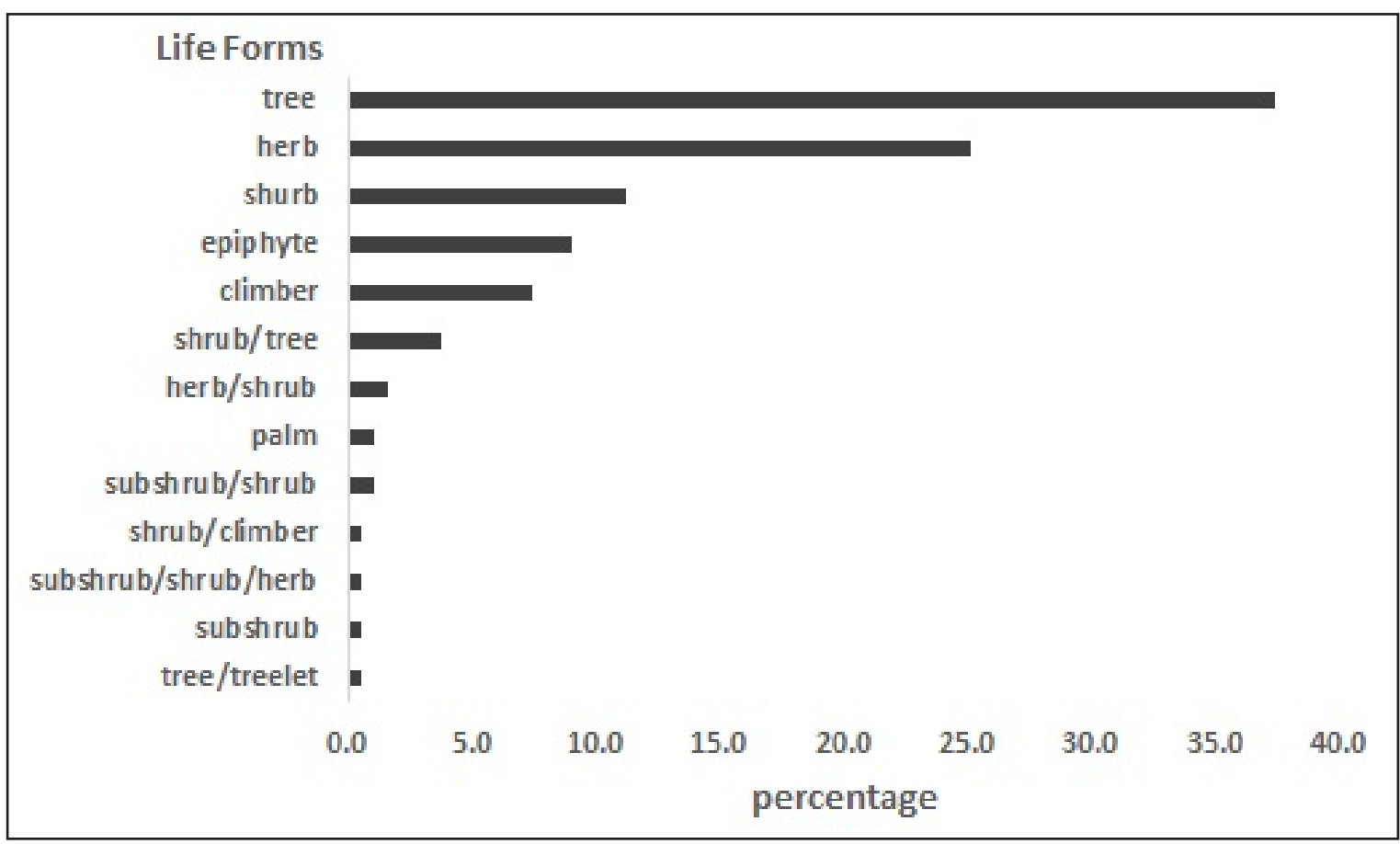

Figure 1. Percentage of species richness for all life forms found in the Ibicatu Ecological Station survey.

Figura 1. Percentual da riqueza de espécies para todas as formas de vida presentes no levantamento florístico da Estação Ecológica de Ibicatu.

We observed that Asplenium formosum was the only fern species in the floristic survey mentioned by the IUCN red list of threatened species (2016), last concern categorized. Regarding angiosperms, we verified that 13 species were classified as endangered or vulnerable in the IUCN red list (Table 1). Cariniana legalis and
Cedrela fissilis are mentioned in the list of endangered species of the state of São Paulo (São Paulo, 2016b), the IUCN red list of threatened species (2016) and the Brazilian Flora Red Book (Martinelli and Moraes, 2013), respectively, as endangered and vulnerable species. Euterpe edulis was classified as endangered in 
the Brazilian Flora Red Book (Martinelli and Moraes, 2013) and vulnerable in the list of endangered species of the state of São Paulo (São Paulo, 2016b). According to the list of endangered species of the state of São Paulo, Peperomia hydrocotyloides was considered extinct in nature and Maytenus ilicifolia was classified as a vulnerable species.

\section{DISCUSSION}

Even though it is a small fragment, the Ibicatu Ecological Station still reports a richness of angiosperm families with SSF traits, as sampled in the previous floristic surveys, which identified 110 (Custódio-Filho et al., 1994) and 103 (Costa and Mantovani, 1995) woody species. From the method used in this survey, which addressed all vascular life forms, 102 species were added, evidencing the importance of preserving this protected area. The families that were richer in species in this survey also mentioned in others SSF floristic woody surveys from the state of São Paulo (Leitão-Filho, 1987; Cardoso-Leite and Rodrigues, 2008; Corrêa et al., 2014; Coelho et al., 2016), which confirmed the forest characteristic formation in the protected area.

About the fern group, the most common families found in this survey were reported in others fern checklists in Brazilian SSF (Melo and Salino, 2002; Forzza et al., 2014; Mazziero and Nonato, 2015), which are among the ten richest fern families in Brazilian flora (Prado et al., 2015). Observing the surveys by these authors, there are few species present in both checklists: Asplenium bradei, Pleopeltis pleopeltifolia, and Doryopteris concolor (Mazziero and Nonato, 2015), Pteris denticulata (Forzza et al., 2014) and Macrothelypteris torresiana (Melo and Salino, 2002). Highlight to Anemia phyllitidis, a species from conserved forest patches (Melo and Salino, 2002) and reported in the checklists by Mazziero and Nonato (2015) and Melo and Salino, 2002.

The fern survey in this small fragment represents $3.7 \%$ of all species in the state of São Paulo (Prado et al., 2015), which we consider as good, due to its total area and the monoculture matrix where it is inserted. The species by total area of the fragment, in hectares (three species), is similar to what was found in studies carried out in larger fragments, such as Forzza et al. (2014) and Mazziero and Nonato (2015), with four and three species per hectare, respectively, sampled in fragments of 200 hectares.
Epiphyte life form represents approximately $10 \%$ of all vascular plants in tropical forest (Gentry and Dodson, 1987), maintaining a fundamental ecological relationship with the local fauna. We verified that 55\% of all epiphytes species found in this survey were also present in other recent checklists (Table 2), carried out in Brazilian SSF fragments in different conservation stages. Tillandsia recurvata demonstrated to be the most generalist species, with occurrence in all surveys, followed by Epiphyllum phyllanthus. Highlight to Peperomia rotundifolia, a very delicate epiphyte species with shaded life, which had been sampled by Marcusso et al. (2016) in SSP of the Porto Ferreira State Park. The presence of this species, considered to be extinct in the state of São Paulo (São Paulo, 2016b), highlights the role of small forest fragments in the conservation of biodiversity (Vidal et al., 2016).

An expressive portion of the species found in this survey refers to herbal life forms ( 47 species), with numbers smaller only than tree life forms. However, few publications about species richness of herb life forms were conducted in semidecidual forests. Observing the survey by Stranghetti and Ranga (1998), we verified that species number was almost four times smaller (10 species). Even so, Adiantum, Commelina and Cyperus genera are present in the two surveys. Anemia phyllitidis is a species common among researches, a common SSF species. Among the herbs, we collected Tradescantia zebrina, a dominant invader species (Zenni and Ziller, 2011) and Zeuxine strateumatica, another exotic species, more common in humid environments in human-modified landscapes (Neto et al., 2011), though it is a non-dominant ruderal species.

Climber life forms are a very important tropical forest component, contributing with 19\% of all species richness in dry and pluvial forests (Gentry and Dodson, 1987). We identified species typical to SSF, sampled in recent checklists in similar forest formations (Udulutsch et al., 2004, 2010; Tibiriçá et al., 2006; Santos et al., 2009). Our sampling was small, considering SSF in the state of São Paulo (Udulutsch et al., 2004, 2010), but this can be attributed to the method used (walking method), and the greater canopy shading and inhibition of the life form growth (Morellato and Leitão-Filho, 1996), because most samples were inside the forest fragment. Future surveys specifically about climbers can add to this life form's species richness. 
Table 2. List of vascular epiphytes also present in other studies conducted in Seasonal Semideciduous Forests. BW: Baú Wood; BBGR: Biological Reserve of Grama Reservoir; ISP: Ibitipoca State Park; NFI: National Forest of Ipanema; SFHS: Santa Fé Hidroeletric Sistem; IMP: Ingá Municipality Park; BG: Botanical Garden of Federal University of Juiz de Fora; PFSP: Porto Ferreira Sate Park; ES: Espírito Santo; MG: Minas Gerais; PR: Paraná; SP: São Paulo; ASSF: Alluvial Seasonal Semideciduous Forest; CER: Cerrado; MSSF: Montane Seasonal Semideciduous Forest; GF: Gallery Forest; SSF: Seasonal Semideciduous Forest

Tabela 2. Lista de epífitas vasculares igualmente presentes em outros estudos realizados na floresta estacional semidecidual. BW: Bosque do Baú; BBGR: Reserva Biológica da Represa do Grama; ISP: Parque Estadual de Ibitipoca; NFI: Floresta Nacional de Ipanema; SFHS: Sistema Hidroelétrico de Santa Fé; IMP: Parque Municipal Ingá; BG: Jardim Botânico da Universidade Federal de Juiz de Fora; PFSP: Parque Estadual de Porto Ferreira; ES: Espírito Santo; MG: Minas Gerais; PR: Paraná; SP: São Paulo; ASSF: Floresta Estacional Semidecidual Aluvial; CER: Cerrado; MSSF: Floresta Estacional Semidecidual Montana; GF: Floresta de Galeria; SSF: Floresta Estacional Semidecidual

\begin{tabular}{|c|c|c|c|}
\hline SPECIES & STUDY AREA & VEGETATION & AUTHORS \\
\hline \multirow[t]{2}{*}{ Aechmea bromeliifolia (Rudge) Baker } & PFSP (SP) & ASSF & Marcusso et al., 2016 \\
\hline & $\mathrm{BW} / \mathrm{BRGR} / \mathrm{ISP}(\mathrm{MG})$ & $\mathrm{CER} / \mathrm{MSSF} / \mathrm{GF}$ & Neto et al., 2009 \\
\hline \multirow[t]{4}{*}{ Epiphyllum phyllanthus (L.) Haw } & NFI (SP) & SSF & Bataghin et al., 2010 \\
\hline & SFHS (ES) & SSF & Couto et al., 2016 \\
\hline & PFSP (SP) & ASSF & Marcusso et al., 2016 \\
\hline & $\mathrm{BW} / \mathrm{BRGR} / \mathrm{ISP}(\mathrm{MG})$ & $\mathrm{CER} / \mathrm{MSSF} / \mathrm{GF}$ & Neto et al., 2009 \\
\hline Microgramma squamulosa (Kaulf.) de & IMP (PR) & SSF & Dettke et al., 2008 \\
\hline \multirow[t]{2}{*}{ la Sota } & PFSP (SP) & ASSF & Marcusso et al., 2016 \\
\hline & $\mathrm{BG}(\mathrm{MG})$ & SSF & Santana et al., 2017 \\
\hline Peperomia rotundifolia (L.) Kunth & PFSP (SP) & ASSF & Marcusso et al., 2016 \\
\hline $\begin{array}{l}\text { Pleopeltis minima (Bory) J. Prado \& } \\
\text { R.Y. Hirai }\end{array}$ & SFHS (ES) & SSF & Couto et al., 2016 \\
\hline Pleopeltis pleopeltifolia (Raddi) Alston & NFI (SP) & SSF & Bataghin et al., 2010 \\
\hline \multirow[t]{3}{*}{ Rhipsalis cereuscula Haw. } & NFI (SP) & SSF & Bataghin et al., 2010 \\
\hline & IMP (PR) & SSF & Dettke et al., 2008 \\
\hline & PFSP (SP) & ASSF & Marcusso et al., 2016 \\
\hline \multirow[t]{5}{*}{ Tillandsia recurvata (L.) L. } & SFHS (ES) & SSF & Couto et al., 2016 \\
\hline & IMP (PR) & SSF & Dettke et al., 2008 \\
\hline & PFSP (SP) & ASSF & Marcusso et al., 2016 \\
\hline & BW/BRGR/ISP (MG) & $\mathrm{CER} / \mathrm{MSSF} / \mathrm{GF}$ & Neto et al., 2009 \\
\hline & $\mathrm{BG}(\mathrm{MG})$ & SSF & Santana et al., 2017 \\
\hline \multirow[t]{3}{*}{ Tillandsia tricholepis Baker } & NFI (SP) & SSF & Bataghin et al., 2010 \\
\hline & IMP (PR) & SSF & Dettke et al., 2008 \\
\hline & PFSP (SP) & ASSF & Marcusso et al., 2016 \\
\hline \multirow[t]{2}{*}{ Tillandsia usneoides (L.) L. } & BW/BRGR/ISP (MG) & $\mathrm{CER} / \mathrm{MSSF} / \mathrm{GF}$ & Neto et al., 2009 \\
\hline & PFSP (SP) & ASSF & Marcusso et al., 2016 \\
\hline
\end{tabular}

Among the sampled species, Dalechampia triphylla, Gouania virgata and Serjania caracasana produce fruits in the dry season (Morellato and Leitão-Filho, 1996). In addition, provision to wildlife throughout the year and serving as potential shelter are other positive aspects of all species sampled in this survey. It is important to emphasize that the Serjania genera can become hyperabundant, especially in places with high incidence of sunlight, considered more degraded (Mello, 2015). According to Mello (2015), Serjania fuscifolia also has hyperabundant potential in more conserved SSF forest patches, requiring attention to be paid to the entire protected area.
Regarding shrubs, 21 typical species sampled in understory sampled reflect friendly microclimatic condition in this small SSF fragment stratum. Actinostemon and Psychotria were sampled in others small semideciduous forests (Ivanauskas et al., 1999; Santos and Kinoshita, 2003; Corrêa et al., 2014), and this reinforces the contribution of this small fragment to the maintenance of species richness in this forest stratum. On the other hand, the historical agricultural use of this area by some Coffea arabica individuals can still be perceived, with exotic species as part of the current understory. 
Our survey for tree and treelet life forms found species of well-conserved forests and very important for forest canopy structures (Durigan et al., 2000; Santos and Kinoshita, 2003), such as Aspidosperma polyneuron, Balfourodendron riedelianum, Cariniana legalis and Cedrela fissilis. In addition, we sampled Euterpe edulis, Trichilia casaretti and T. silvatica, species important for wildlife food resource, preserving network interaction (Dias et al., 2016; Beca et al., 2017), as well as ecosystem services (Turner et al., 2007), and maintaining gene flow (Martins et al., 2016). However, Tecoma stans is considered an aggressive exotic species (Renó et al., 2007; Silva et al., 2008), requiring attention due to negative impact on native species.

The Ibicatu Ecological Station is a protected area since 1987, inserted in human-modified landscape, and $17.5 \%$ of the original forest coverage is composed of small fragments (São Paulo, 2016a). Nevertheless, it presents important and typical species of the SSF forest, some of them threatened, distributed in tree life forms (Aspidosperma polyneuron, Balfourodendron riedelianum, Cariniana legalis, Cedrela fissilis, Esenbeckia leiocarpa, Maytenus ilicifolia and Trichilia silvatica), palm life forms (Euterpe edulis), climber life forms (Cissampelos pareira) and herb life forms (Peperomia hydrocotyloides). Maytenus ilicifolia and Peperomia hydrocotyloides are mentioned in the list of endangered species of the state of São Paulo (São Paulo, 2016b) as species presumably extinct in the State, which increases the relevance of this small protected area.

In contrast, we sampled exotic invasive species (Renó et al., 2007; Silva et al., 2008; Zenni and Ziller, 2011), of genera with hyperabundance potential (Mello, 2015), characteristic of an area that suffers anthropic pressures. The balance found between the number of tree and non-tree species still is consistent with the forest dynamics, but it becomes necessary to isolate the area, to protect it from the negative impacts of the surroundings. Otherwise, conservation status can be altered, from increasingly serious and regular negative impacts. Future studies based on this research should be conducted to continuously conserve and increase scientific knowledge about this protected area.

\section{CONCLUSIONS}

We presented a checklist update of the Ibicatu Ecological Station vascular flora, adding 102 species to the previously published checklist, with woody and non-woody life forms. We consider that this small forest fragment presents rich vascular flora, typical of the SSF of the São Paulo State countryside, some of them threatened species. Our results evidence and corroborate the extreme relevance of this small SSF fragment for local and regional biodiversity.

\section{REFERENCES}

BATAGHIN, F.A.; BARROS, F.; PIRES, J.S.R. Distribuição da comunidade de epífitas vasculares em sítios sob diferentes graus de perturbação na Floresta Nacional de Ipanema, São Paulo, Brasil. Revista Brasileira de Botânica, v. 33, n. 3, p. 501-512, 2010. http://dx.doi.org/10.1590/S0100-84042010000300012.

BECA, G. et al. High mammal species turnover in forest patches immersed in biofuel plantations. Biological Conservation, v. 210, p. 352-359, 2017. http://dx.doi.org/10.1016/j.biocon.2017.02.033.

BENÍTEZ-MALVIDO, J.; MARTÍNEZ-RAMOS, M. Impact of forest fragmentation on understory plant species richness in Amazonia. Conservation Biology, v. 17, n. 2, p. 389-400, 2003. http://dx.doi. org/10.1046/j.1523-1739.2003.01120.x.

BRANCALION, P.H.S.; RODRIGUES, R.R., GANDOLFI, S. Restauração florestal. São Paulo, SP: Oficina de Textos, 2015. 432 p.

COELHO, S. et al. Composição florística e caracterização sucessional como subsídio para conservação e manejo do PNMCBIO, Sorocaba - SP. Ciência Florestal, Santa Maria, v. 26, n. 1, p. 331-344, 2016

CORRÊA, L.S. et al. Estrutura, composição florística e caracterização sucessional em remanescente de Floresta Estacional Semidecidual no Sudeste do Brasil. Revista Árvore, Viçosa, v. 38, n. 5, p. 799-809, 2014. COSTA, L.G.S.; MANTOVANI, W. Flora arbustivoarbórea de trecho de mata mesófila semidecídua, na Estação Ecológica de Ibicatu, Piracicaba (SP). Hoehnea, v. 22, n. 1, p. 47-59, 1995. 
COUTO, D.R. et al. Epífitas vasculares em floresta estacional Semidecidual no estado do Espírito Santo e similaridade com outras florestas estacionais no Leste do Brasil. Acta Scientiarum, v. 38, n. 2, p. 169-177, 2016.

CUSTÓDIO-FILHO, A. et al. Composição florística da vegetação arbórea da floresta mesófila semidecídua da estação ecológica de Ibicatu, Piracicaba, SP. Revista do Instituto Florestal, v. 6, p. 99-111, 1994.

DETTKE, G.A.; ORFRINI, A.C.; GUTIERRE, M.A.M. Composição florística e distribuição de epífitas vasculares em um remanescente alterado de floresta estacional Semidecidual no Paraná, Brasil. Rodriguésia, v. 59, n. 4, p. 859-872, 2008. http:// dx.doi.org/10.1590/2175-7860200859414.

DIAS, D.F.C. et al. Beauty before age: landscape factors influence bird functional diversity in naturally regenerating fragments, but regeneration age does not. Restoration Ecology, v. 24, n. 2, p. 259-270, 2016. http://dx.doi.org/10.1111/rec.12293.

DURIGAN, G. et al. Estrutura e diversidade do componente arbóreo da floresta na Estação Ecológica dos Caetetus, Gália, SP. Revista Brasileira de Botânica, v.23, n. 4, p.371-383. 2000.

Control of invasive plants: ecological and socioeconomic criteria for the decision making process. Natureza \& Conservação, v. 11, n. 1, p. 23-30, 2013. http://dx.doi.org/10.4322/natcon.2013.003.

FILGUEIRAS, T.S. et al. Caminhamento: um método expedito para levantamentos florísticos qualitativos. Cadernos de Geociências, v. 12, p. 39-43, 1994.

FLORA OF BRAZIL 2020 in construction. Jardim Botânico do Rio de Janeiro. Available at: $<$ http:// floradobrasil.jbrj.gov.br>. Access on: 01 Oct. 2017.

FORZZA, R.C. et al. Flora vascular da reserva biológica da represa do Grama, Minas Gerais e sua relação florística com outras florestas do sudeste brasileiro. Rodriguésia, v. 65, n. 2, p. 275-292, 2014. http:// dx.doi.org/10.1590/S2175-78602014000200001.

GENTRY, A.H.; DODSON, C.H. Diversity and biogeography of neotropical vascular epiphytes. Annals of the Missouri Botanical Garden, v. 74, n. 2, p. 205-233, 1987. http://dx.doi.org/10.2307/2399395.
INSTITUTO BRASILEIRO DE GEOGRAFIA E ESTATÍSTICA - IBGE. Manual Técnico da Vegetação Brasileira. 2. ed. Rio de Janeiro: IBGE, 2012. 271 p. (Série Manuais Técnicos em Geociências, n. 1). Available at: <http://www.ibge.gov.br/home/geociencias/ recursosnaturais/vegetacao/manual_vegetacao.shtm>. Access on: 15 June 2016.

INSTITUTOS NACIONAIS DE CIÊNCIA E TECNOLOGIA - INCT. Herbário Virtual da Flora e dos Fungos. Available at: <http://inct.splink.org. br/>. Access on: Access on: 15 June 2016.

INTERNATIONAL UNION FOR CONSERVATION OF NATURE - IUCN. Red List of Threatened Species 2015. Available at: $<$ http://www.redlist.org $>$. Access on: 31 June 2016.

IVANAUSKAS, N.M. et al. Fitossociologia de um trecho de floresta estacional semidecidual em Itatinga, São Paulo, Brasil. Scientia Forestalis, v. 56, p. 83-99, 1999.

KÖEPPEN, W. Climatologia: con un estúdio de los climas de la Tierra. Mexico City: Fondo de Cultura Economica, 1948, 478 p.

LAURENCE, W.F. et al. Habitat fragmentation, variable edge effects, and the landscape-divergence hypothesis. Plos One, v. 2, n. 10, p. 1-8, 2007.

LEÃO, J.F.M.C. Análise de uma unidade de conservação ameaçada - a estação ecológica de Ibicatu, Piracicaba (SP) como subsídio para elaboração de seu plano de manejo. 1994. $178 \mathrm{f}$. Dissertation (Master's Forest Science). Escola Superior de Agricultura "Luiz de Queiroz", Universidade de São Paulo, Piracicaba. Available at: http://www.teses. usp.br/. Access on: 15 July 2016.

LEITE, E.C.; RODRIGUES, R.R. Fitossociologia e caracterização sucessional de um fragmento de Floresta Estacional no Sudeste do Brasil. Revista Árvore, v. 32, n. 3, p. 583-595, 2008. http://dx.doi. org/10.1590/S0100-67622008000300019.

LEITÃO-FILHO, H.F. Considerações sobre a florística de florestas tropicais e subtropicais do Brasil. Circular Técnica IPEF, v. 35, p. 41-46, 1987.

MARCUSSO, et al. Epífitas vasculares do Parque Estadual de Porto Ferreira, São Paulo, Brasil. Revista Instituto Florestal, v. 28, n. 2, p. 119-133, 2016. 
CORRÊA, L.S. et al. Ibicatu vascular flora.

MARTINELLI, G.; MORAES, M.A. (Org.). Livro vermelho da flora do Brasil. Rio de Janeiro: Instituo de Pesquisas Jardim Botânico do Rio de Janeiro. 2013, 1100 p.

MARTINS, K. et al. The role of very small fragments in conserving genetic diversity of a common tree in a hyper fragmented Brazilian Atlantic forest landscape. Conservation Genetics, v. 17, n. 3, p. 509-520, 2016. http://dx.doi.org/10.1007/s10592-015-0800-7.

MAZZIERO, F.F.; NONATO, F.R. Ferns and lycophytes from Jaú, São Paulo, Brazil. Check List, v. 11, n. 6, p. 1-10, 2015. http://dx.doi.org/10.15560/11.6.1798.

MELLO, F.N.A. Padrões da comunidade de trepadeiras e suas relações coma biomassa arbórea e a regeneração natural em uma floresta estacional semidecidual em Piracicaba, SP: implicações no manejo de fragmentos florestais degradados. 2015. 105 f. Dissertation (Master's Forest Science). Escola Superior de Agricultura "Luiz de Queiroz", Universidade de São Paulo, Piracicaba. Available at: $<$ http://www. teses.usp.br/>. Access on: 01 Oct. 2017.

MELO, L.C.N.; SALINO, A. Pteridófitas de duas áreas de floresta da bacia do rio Doce no estado de Minas Gerais, Brasil. Lundiana, v. 2, n. 2, p. 129-139, 2002.

MITTERMEIER, R.A. et al. Hotspots revisited: earth's biologically richest and most endangered terrestrial ecoregions. Mexico City: CEMEX, 2004. 392 p.

MORELLATO, P.C.; LEITÃO-FILHO, H. Reproductive phenology of climbers in a Southeastern Brazilian Forest. Biotropica, v. 28, n. 2, p. 180-191, 1996. http://dx.doi.org/10.2307/2389073.

MORO, M.F. et al. Alienígenas na sala: o que fazer com espécies exóticas em trabalhos de taxonomia, florística e fitossociologia? Acta Botânica Brasílica, v. 26, n. 4, p. 991-999, 2012.

MYERS, N. Biodiversity hotspots revisited. Bioscience, v. 53, n. 10, p. 916-917, 2003. http:// dx.doi.org/10.1641/0006-3568(2003)053[0916:BH $\mathrm{R}] 2.0 . \mathrm{CO} ; 2$.

NETO, L.M.; FORZZA, R.C.; ZAPPI, D. Angiosperm epiphytes as conservation indicators in forest fragments: a case study from southeastern Minas Gerais, Brazil. Biodiversity and Conservation, v. 18, n. 14, p. 3785-3807, 2009. http://dx.doi.org/10.1007/s10531009-9679-2.
NETO, L.M.; RODRIGUES, M.M.; CRUZ, D. Zeuxine strateumatica (Orchidaceae) goes south: a first record for Brazil. Kew Bulletin, v. 66, n. 1, p. 155-158, 2011. http://dx.doi.org/10.1007/s12225-011-9271-2.

PIRACICABA. Secretaria de Defesa do Meio Ambiente. Unidades de Conservação. Available at: $<$ http:// sedema.wixsite.com/sedema/unidades-de-conservao>. Access on: 12 Abril 2017.

PRADO, J. et al. Diversity of ferns and licophytes in Brazil. Rodriguésia, v. 66, n. 4, p. 1073-1083, 2015. http://dx.doi.org/10.1590/2175-7860201566410.

RENÓ, L.R.; MOSCHETA, I.S.; BRACCINI, A.L. Morfo-anatomia de fruto e semente do amarelinho (Tecoma stans - Bignoniaceae). Revista Brasileira de Sementes, v. 29, n. 3, p. 18-30, 2007. http://dx.doi. org/10.1590/S0101-31222007000300003.

RICHARDSON, D.M. et al. Blackwell Science, Ltd Naturalization and invasion of alien plants: concepts and definitions. Diversity \& Distributions, v. 6, n. 2, p. 93-107, 2000. http://dx.doi.org/10.1046/j.14724642.2000.00083.x.

RODRIGUES, R.R. A vegetação de Piracicaba e municípios do entorno. Circular técnica IPEF, v. 189, p. 1-20, 1999.

SANTANA, L.D. et al. Diversity, vertical structure and floristic relationships of vascular epiphytes in na urban remnant of the Brazilian Atlantic Forest. Hoehnea, v. 44, n. 1, p. 123-138, 2017. http://dx.doi. org/10.1590/2236-8906-57/2016.

SANTOS, K.; KINOSHITA, L.S. Flora arbustivo arbórea do fragmento de floresta estacional semidecidual do Ribeirão Cachoeira, município de Campinas, SP. Acta Botanica Brasílica, v. 17, n. 3, p. 325-341, 2003. http://dx.doi.org/10.1590/S0102-33062003000300001.

SANTOS, K.; KINOSHITA, L.S.; REZENDE, A.A. Composição florística de trepadeiras em florestas sazonais semideciduais do sudeste do Brasil. Biota Neotropica, v. 9, n. 4, p. 175-188, 2009. http://dx.doi. org/10.1590/S1676-06032009000400018.

SÃO PAULO (Estado). Secretaria de Meio Ambiente do Estado (SMA). Inventário florestal do estado de São Paulo: mapa do município de Piracicaba. São Paulo, 2016. Available at: $<$ http://s.ambiente.sp.gov. br/sifesp/piracicaba.pdf $>$ Access on: 17 June 2016a. 
SÃO PAULO (Estado). Secretaria do Meio Ambiente (SMA). Resolução no 57, de 05 de junho de 2016. Publica a segunda revisão da lista oficial das espécies da flora ameaçadas de extinção no Estado de São Paulo. Diário Oficial do estado de São Paulo, Poder Executivo, v. 127, n. 46, 7 jun. de 2016b. Seção I, p. 69-71.

Secretaria de Meio Ambiente do Estado SMA. Fundação para a Conservação e a Produção Florestal do Estado de São Paulo - Fundação Florestal. Available at: <http://fflorestal.sp.gov.br/ institucional/missao/>. Access on: 01 Oct. 2017.

SILVA, J.A.; REIS, T.E.S.; REIS, L.C. Análise da infestação do amarelinho (Tecoma stans) na zona rural do município de Bandeirantes - PR. Semina: Ciências Agrárias, v. 29, n. 1, p. 83-92, 2008. http:// dx.doi.org/10.5433/1679-0359.2008v29n1p83.

SMITH, A.R. et al. A classification for extant ferns. Taxon, v. 55, n. 3, p. 705-731, 2006. http://dx.doi. org/10.2307/25065646.

SPECIESLINK DATABASES. 2016. Available at: $<$ http://splink.cria.org.br/tools?criaLANG=en $>$. Access on: 15 July 2016.

STRANGHETTI, V.; RANGA, N.T. Levantamento florístico das espécies vasculares da floresta estacional mesófila semidecídua da Estação Ecológica de Paulo de Faria, SP. Revista Brasileira de Botanica. Brazilian Journal of Botany, v. 21, n. 3, p. 1-16, 1998. http:// dx.doi.org/10.1590/S0100-84041998000300008.

THE ANGIOSPERM PHYLOGENY GROUP. An update of the Angiosperm Phylogeny Group classification for the orders and families of flowering plants: APG IV. Botanical Journal of the Linnean Society, v. 16, p. 105-121, 2016.

TIBIRIÇÁ, Y.J.A.; COELHO, L.F.M.; MOURA, L.C. Florística de lianas em um fragmento de floresta estacional semidecidual, Parque Estadual de Vassununga, Santa Rita do Passa Quatro, SP, Brasil. Acta Botanica Brasílica, v. 20, n. 2, p. 339-346, 2006. http://dx.doi. org/10.1590/S0102-33062006000200009.

TROPICOS. 2016. Available at: <http://www.tropicos. org >. Access on: 15 July 2016.

TURNER, W.R. et al. Global conservation of biodiversity and ecosystem services. Bioscience, v. 57, n. 10, p. 868-873, 2007. http://dx.doi.org/10.1641/B571009.
UDULUTSCH, R.G.; ASSIS, M.A.; PICCHI, D.G. Florística de trepadeiras numa floresta estacional semidecídua, Rio Claro - Araras, estado de São Paulo, Brasil. Revista Brasileira de Botanica, v. 27, n. 1, p. 125-134, 2004. http://dx.doi.org/10.1590/S010084042004000100014.

UDULUTSCH, R.G. et al. Composição florística e chaves de identificação para lianas da Estação Ecológica dos Caetetus, estado de São Paulo, Brasil. Rodriguésia, v. 61, n. 4, p. 715-730, 2010. http:// dx.doi.org/10.1590/2175-7860201061412.

VIANA, V.M.; PINHEIRO, L. Conservação da biodiversidade em fragmentos florestais. Série técnica IPEF, v. 12, p. 25-42, 1998.

VIDAL, C.Y. et al. Biodiversity conservation of forests and their ecological restoration in highly-modified landscapes. In: GHELER-COSTA, C.; LYRA-JORGE, M.C.; VERDADE, L.M. (Eds.) Biodiversity in agricultural landscapes of Southeastern Brazil. Warsaw: De Gruyter Open Ltd: Berlin: Part of Walter de Gruyter GmbH, 2016. 334 p. http://dx.doi. org/10.1515/9783110480849-010.

WALTER, B.M.T.; GUARINO, E.S.G. Comparação do método de parcelas com o "levantamento rápido" para amostragem da vegetação arbórea do Cerrado sentido restrito. Acta Botanica Brasílica, v. 2, n. 20, p. 285-297, 2006. http://dx.doi.org/10.1590/S010233062006000200005.

WANDERLEY, M.G.L. et al. Flora Fanerogâmica do Estado de São Paulo. São Paulo: HUCITEC, 2002. v. 2, 391 p. Available at: <http://botanica.sp.gov.br/ ffesp_online/>. Access on: 15 July 2016.

. Flora Fanerogâmica do Estado de São Paulo. São Paulo: FAPESP/RiMa, 2003. v. 3, 367 p. Available at: $<$ http://botanica.sp.gov.br/ffesp_online/ $>$. Access on: 15 July 2016.

Flora Fanerogâmica do Estado de São Paulo. São Paulo: FAPESP/RiMa, 2005. v. 4, 392 p. Available at: $<$ http://botanica.sp.gov.br/ffesp_online/>. Access on: 15 July 2016.

ZENNI, R.D.; ZILLER, S.R. An overview of invasive plants in Brazil. Revista Brasil. Bot, v. 34, n. 3, p. 431-446, 2011. 